\title{
Effect of deep brain simulation on arm, leg, and chin tremor in Parkinson disease
}

\author{
Kalman Katlowitz, PhD, Mia Ko, MD, Alon Y. Mogilner, MD, PhD, and Michael Pourfar, MD \\ Center for Neuromodulation, Department of Neurosurgery, New York University Langone Medical Center, New York, New York
}

OBJECTIVE The efficacy of deep brain stimulation (DBS) of the subthalamic nucleus (STN) in the treatment of Parkinson disease (PD)-related tremor has been well established. However, the relative impact on arm, leg, and chin tremor has been less clearly elucidated. The authors evaluated the distribution of tremors in a PD cohort undergoing STN DBS and sought to evaluate the differential impact of DBS as a function of tremor location.

METHODS A retrospective study of patients with PD with tremor who underwent DBS surgery between 2012 and 2016 was performed to evaluate the impact of STN stimulation on overall and regional tremor scores.

RESULTS Across 66 patients the authors found an average of $78 \%$ overall reduction in tremor after 6 months. In this cohort, the authors found that tremor reduction was somewhat better for arm than for leg tremors, especially in instances of higher preoperative tremor ( $84 \%$ vs $71 \%$ reduction, respectively, for initial tremor scores $\geq 2$ ). No significant difference in response was found between patients with medication-responsive versus medication-nonresponsive tremors.

CONCLUSIONS The authors found that although DBS improved tremor in all regions, the improvement was not uniform between chin, arm, and leg_even within the same patient. The reasons behind these differing responses are speculative but suggest that STN DBS may more reliably reduce arm tremors than leg tremors.

https://thejns.org/doi/abs/10.3171/2018.7.JNS18784

KEYWORDS deep brain stimulation; tremor; Parkinson disease; functional neurosurgery

$\mathrm{D}$ EEP brain stimulation (DBS) of the subthalamic nucleus (STN) has become a part of the standard of care in the management of select patients with Parkinson disease (PD) since its introduction in the

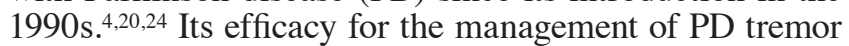
in particular has been repeatedly demonstrated in several large cohorts with reductions often on the order of $75 \%-$ $90 \%{ }^{3,11}$ This consistent improvement, however, is often reported as an aggregate result, with less attention directed to the impact of stimulation specifically on chin, arm, and leg tremor, all of which can be variably affected in PD even in the same patient. We performed a retrospective chart review of all patients with PD who were evaluated between 2012 and 2016 and who underwent STN DBS largely if not exclusively to improve tremors. We assessed overall tremor improvement with additional focus on the response for different regions of the body, and then analyzed whether there was any correlation in improvement between one region and another within the same patient.

\section{Methods}

A retrospective study was performed in patients who underwent DBS surgery to treat the symptoms of pharmacologically intractable PD. We reviewed the charts of patients with PD who underwent DBS at the New York University Langone Medical Center for Neuromodulation between January 1, 2012, and December 31, 2016. Of these, we examined the results for patients who had a preoperative tremor score in one limb of at least 1 on the Unified Parkinson's Disease Rating Scale part III (UPDRS-III). Patients without at least 6 months of postoperative followup were excluded. Medication-refractory tremor was defined as reported by the patient and/or as generally reflected by minimal change in the tremor scores with levodopa in the "on" versus the "off" mode in those patients who continued to take levodopa for other responsive symptoms.

For a baseline metric, we used the UPDRS-III subscores obtained in the "medication off" state prior to surgery. The

ABBREVIATIONS DBS = deep brain stimulation; PD = Parkinson disease; STN = subthalamic nucleus; UPDRS = Unified Parkinson's Disease Rating Scale.

SUBMITTED March 20, 2018. ACCEPTED July 3, 2018.

INCLUDE WHEN CITING Published online December 7, 2018; DOI: 10.3171/2018.7.JNS18784. 
most recent UPDRS-III tremor subscores post-DBS, in the "stimulation on"/"medication off" state, were collected as the outcome for these patients. Data from all 4 limbs and chin were included in subjects with bilateral lead implantation. If the subject received a unilateral implant, then we only included data from the chin and contralateral limbs. Subjects who had not yet reached the 6-month post-DBS time point or who lacked a post-6-month "medication off"/"stimulation on" tremor score were excluded. Within subjects, limbs with baseline scores of 0 were likewise excluded from analysis.

Nonparametric hypothesis testing (e.g., Wilcoxon signed-rank test) was used for most of the analysis, reflecting the ordinal nature of the UPDRS scores. Limbs from contralateral hemispheres within the same subjects were considered statistically independent unless otherwise noted. To directly compare the impact of DBS across limbs, a general linear model was implemented in which categorical regressors were used to predict change in tremor scores relative to baseline. We used sampling weights to account for bias in initial scores.

Subjects were not compensated or rewarded for participating in this review. Approval was acquired from our institutional review board.

\section{Results \\ Data Set}

We reviewed the records of 152 patients with PD who underwent STN DBS at a single center between 2012 and 2016. Of these, 66 patients cited tremor as a prominent or bothersome symptom and returned for follow-up after 6 months. The demographic data for these patients are presented in Table 1 along with their programming parameters. More than half (39/66) responded to medical treatment before implantation. The average time to DBS for medication-responsive tremors was $9.8 \pm 3.8$ years as compared with medication-refractory tremors, which averaged $6.6 \pm 2.9$ years. Notably, we found strong differences in the location of the tremor across extremities: whereas $98 \%$ of subjects with tremor-predominant PD showed at least some level of arm tremor, only $77 \%$ showed leg tremor and $47 \%$ showed chin tremor. This divide is even more obvious for severe tremors (preoperative tremor score of 4): whereas $36 \%$ of patients had severe arm tremor, only $8 \%$ and $2 \%$ of patients showed severe leg or chin tremor, respectively. Isolated arm tremor or leg tremor could be found in $30 \%$ of cases $(n=104$ ipsilateral sides, 25 with only arm tremor and 6 with only leg tremor), although when both tremors were present the severity scores were positively correlated $(n=73$ sides; $r=0.30, p$ $=0.01$, Spearman's rank correlation). Of these 66 subjects, 42 received bilateral implants, 20 received implants only in the left STN, and 4 received implants only in the right STN (Table 1). After excluding limbs with initial UPDRSIII scores of 0 , our data set consisted of pre- and post-DBS tremor scores of 98 arms, 79 legs, and 31 chins.

\section{Effect of DBS on Tremor Score}

DBS broadly improved tremor associated with PD, reducing mean UPDRS scores from 2.4 to 0.5 ( $\mathrm{p}<0.001$,
TABLE 1. Demographic data in 66 patients with PD

\begin{tabular}{lc}
\hline \multicolumn{1}{c}{ Characteristic } & Value \\
\hline Patient data & \\
\hline Sex & 50 \\
\hline Male & 16 \\
\hline Female & 39 \\
\hline Levodopa responsive & 27 \\
\hline Yes & $61.9 \pm 9.28$ \\
\hline No & $8.5 \pm 3.8$ \\
\hline Age in yrs & \\
\hline No. of yrs w/ PD & 45 \\
\hline Stimulation parameter $(n=108)$ & 63 \\
\hline Polarity & 68 \\
\hline Monopolar & 40 \\
\hline Bipolar & $2.94 \pm 0.83$ \\
\hline Electrodes & $3 \pm 1.4$ \\
\hline Single & $79.8 \pm 21$ \\
\hline Multiple & $154 \pm 27$ \\
\hline Voltage $(n=93)$ & \\
\hline Amperage $(n=15)$ & \\
\hline pressed as the number of patients, number of electrodes, or as the mean \pm SD. \\
\hline PW ( $\mu$ sec $)$ \\
\hline Frequency $(\mathrm{Hz})$
\end{tabular}

Wilcoxon signed-rank test) in limbs and chins with preoperative tremor, with a mean of $78 \%$ improvement on original scores. The average effect of DBS on tremor in individual regions was further analyzed and demonstrated significant improvement in chin, arm, and leg ( $\mathrm{p}<0.001$, Wilcoxon signed-rank test) (Fig. 1, Table 2). We next looked at the impact of DBS on tremor as a function of initial tremor score and found that DBS of the STN improved the average tremor scores across all initial tremor magnitudes $(\mathrm{p}<0.001$, Wilcoxon signed-rank test for each level individually) and was independent of whether or not medication alone was initially sufficient to control tremors $(\mathrm{p}<0.001$, Wilcoxon signed-rank test for each group separately). Despite the correlation in initial tremor scores reported above, we found no correlation in arm and leg tremor responses to DBS for ipsilateral sides with initial tremor scores of $\geq 2$ ( $n=38 ; p>0.5$, Spearman's rank correlation), even when the initial scores were identical $(\mathrm{n}=$ $6 ; p>0.5$, Spearman's rank correlation).

\section{Nonresponders}

A clear majority of patients improved with DBS, with 92/98 (94\%) of arms, 68/79 (86\%) of legs, and 29/31 (94\%) of chins responding by $\geq 25 \%$. However, despite the obvious average benefit of DBS, this robust improvement did not accrue equally to all patients. To examine the probability of a response, we defined a nonresponsive limb as one with $<25 \%$ improvement in its UPDRS-III score. 

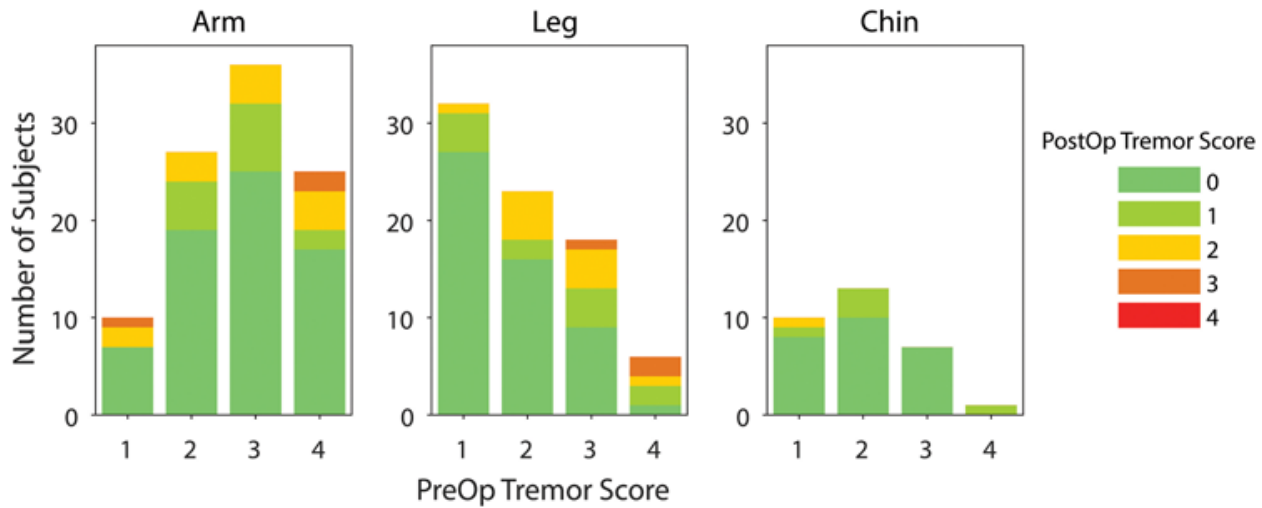

FIG. 1. Bar graphs showing effect of DBS on tremor scores. The distribution of tremor scores after DBS surgery is displayed as a function of preoperative scores for each tremor type. Colors indicate the tremor scores in the "stimulation on" condition.

In 73 unilateral pairs of limbs that had pre-DBS tremor we found that 3 did not improve in either extremity after DBS surgery, which was a larger number than expected by chance $(\mathrm{p}<0.001$, chi-square test for independence). Lead placement was analyzed for all 3 subjects and appeared to be within normal stereotactic limits with one possible exception (Table 3).

\section{Arm Versus Leg}

Throughout our analyses we noted that although leg scores tended to improve, they did so at a lower rate than arm scores. We directly examined the subjects with the same initial scores in ipsilateral arm and leg, noting that, on average, each subject's leg tremor improved only $80 \%$ relative to the improvement in arm tremor. Although this difference was not significant, this is probably due to our lower power for subjects that fit this criterion $(\mathrm{n}=11$; $\mathrm{p}$ $=0.25$, paired Wilcoxon signed-rank test). We therefore next quantified the magnitude of this effect. As indicated above, arms were approximately $10 \%$ more likely to respond to DBS than legs. Although this ratio did not reach significance ( $\mathrm{p}=0.07,1$-sided Fisher's exact test), if we restrict our data set to those with a larger initial tremor (defined as initial scores of $\geq 2$ ), then we do find that arms are more likely to improve than legs ( $p<0.05,1$-sided Fisher's exact test). Additionally, we found that arm scores improved by a significantly larger amount than leg scores (decrease in tremor score of 2.3 vs 1.4 , respectively; $\mathrm{p}<$ 0.001 , Wilcoxon rank-sum test). However, these results could be biased by the fact that arms tended to have worse pre-DBS scores (Fig. 1), leaving more room for improvement. We therefore redid our analysis in two ways. First, we noted that the mean improvement was greater in arm tremor in every baseline level above 1 . However, probably due to our lower sample size after splitting our data set, this effect only reached significance for initial tremor score of 4 ( $p<0.05$ for initial score of $4, p>0.1$ for all others, Wilcoxon rank-sum test). To increase the power of our analysis, we reanalyzed all the levels together, accounting for our bias by weighting our data to the relative probability of the starting level, and found that arm improved more than leg ( $p<0.05$, t-test on general linear model coefficient). Interestingly, despite these results, leg and arm tend to have final scores in similar ranges $(p>0.5$, Wilcoxon rank-sum test), probably because initial leg tremor was usually not as severe as initial arm tremor.

\section{Levodopa-Resistant Tremor}

We compared the impact of DBS on the tremor of subjects who did not respond to levodopa with its impact on those whose tremor was levodopa responsive..$^{10,13}$ Despite the differences in their pharmacological treatment response, there was no difference in the response to DBS, with both groups exhibiting similar tremor scores in the "stimulation on"/"medication off" condition ( $p>0.5$, Wilcoxon rank-sum test) in arm, leg, and chin tremors, and similar improvement relative to baseline ( $p>0.2$, t-test on general linear model coefficient).

\section{Discussion}

Similarly to other studies, our single-center experience demonstrated that DBS of the STN significantly improves PD-associated tremor irrespective of the patient's preoperative tremor response to medications. The mean tremor reduction of $78 \%$ is in line with prior reports of ranges between $40 \%$ and $90 \%$ reduction. ${ }^{5,9,14,18,19,22,23,25,30,33}$ However, most of these studies report tremor either as part of an overall motor improvement, or as an aggregate tremor score. ${ }^{26}$ This summary information alone does not fully elucidate the nature of the tremor outcomes with respect to midline, upper-extremity, and lower-extremity responsiveness. Grouping these scores assumes that they all reflect a single process, yet upper- and lower-extremity circuitry may respond differently to the general severity of the par-

TABLE 2. Average UPDRS outcomes after DBS surgery

\begin{tabular}{crrrrrrr}
\hline & & \multicolumn{2}{c}{ Preop Motor Score } & & \multicolumn{2}{c}{ Postop Motor Score } \\
\cline { 7 - 8 } \cline { 6 - 7 } Variable & No. & Mean & SD & & Mean & SD \\
\hline All & 208 & 2.4 & 1.0 & & 0.5 & 0.8 \\
\hline Arm & 98 & 2.8 & 0.9 & & 0.5 & 0.8 \\
\hline Leg & 79 & 2.0 & 1.0 & & 0.5 & 0.9 \\
\hline Chin & 31 & 2.0 & 0.8 & & 0.2 & 0.5 \\
\hline
\end{tabular}


TABLE 3. Data on nonresponders

\begin{tabular}{|c|c|c|c|}
\hline Factor & Case 1 & Case 2 & Case 3 \\
\hline \multicolumn{4}{|l|}{ Coordinates } \\
\hline \multicolumn{4}{|l|}{ Lt Lead, contact 0} \\
\hline Lateral & 11.9 & 12.3 & 11.3 \\
\hline Posterior & 3.2 & 5.1 & 3.7 \\
\hline Inferior & 4.5 & 6.4 & 5.3 \\
\hline \multicolumn{4}{|l|}{ Rt Lead, contact 0} \\
\hline Lateral & 10.6 & 9.8 & 11.3 \\
\hline Posterior & 3.7 & 4.6 & -4.7 \\
\hline Inferior & 4.3 & 5.4 & -5.1 \\
\hline Lead associated w/ less response & Rt \& It & $\mathrm{Rt}$ & $\mathrm{Rt}$ \\
\hline \multicolumn{4}{|l|}{ LEDD } \\
\hline Preop & $1800 \mathrm{mg}$ & $350 \mathrm{mg}+$ amantadine $300 \mathrm{mg}$ & 1150 mg + apomorphine \\
\hline Postop & $1200 \mathrm{mg}$ & $300 \mathrm{mg}$ & $550 \mathrm{mg}$ \\
\hline \multicolumn{4}{|l|}{ Motor UPDRS score (meds off/on) } \\
\hline Preop & $26 / 13$ & $37 / 23$ & $30 / 7$ \\
\hline Postop* & $33 / 14$ & $8 / 6$ & $8 / 4$ \\
\hline Comments & $\begin{array}{l}\text { No improvement in } \\
\text { tremor or dyskine- } \\
\text { sias. Revision recom- } \\
\text { mended. }\end{array}$ & $\begin{array}{l}\text { Significant tremor reduction but still emerged during walking. } \\
\text { Less-effective lead intentionally positioned more medially } \\
\text { based on intraop impression of superior tremor control. }\end{array}$ & $\begin{array}{l}\text { Further tremor control } \\
\text { limited by stimulation- } \\
\text { induced dyskinesias. }\end{array}$ \\
\hline
\end{tabular}

LEDD = levodopa-equivalent daily dose; meds = medications.

Coordinates of the leads are given in relation to the anterior commissure-posterior commissure (AC-PC) base system and reflect the geometric center of the lowest contact, as determined on postoperative stereotactic CT scans fused to preoperative MR images. We also provide the medication regimen (LEDD, per Tomlinson et al.) and the motor scores in the "medication on" condition.

* "Stimulation on" condition.

kinsonian state. Indeed, we found that these tremors can occur independently of each other and can have individual responses to DBS, supporting the idea that tremor relates to more than simple nigral degeneration. ${ }^{17}$

This retrospective review sought to better understand the impact of STN stimulation on rest tremors affecting different body regions and is, to our knowledge, the largest cohort to look at regional tremor response. Viewing STN DBS for PD through this more specific lens also offered an opportunity to better characterize the baseline characteristics of patients with tremor-predominant PD compared with other PD patients pursuing surgery at a single center over several years. We found that $59 \%$ of all patients undergoing DBS for PD had tremor-predominant disease compared with $41 \%$ without tremors (i.e., akinetic-rigid variant) and that within the tremor cohort, 59\% were medication responsive versus $41 \%$ who were not. Interestingly, outcomes were similar for both groups. However, not surprisingly, the time to DBS was shorter for the medication-nonresponsive group by an average of 3 years (although with significant overlap in their distributions).

The large sample size of our data set enabled us to evaluate the effect of DBS on tremors in less commonly affected areas, such as the leg and chin. While we were unable to identify any publications reporting prevalence of chin or leg tremor in a DBS cohort by using related MEDLINE search terms, our data show that tremor in either leg or chin may affect more than three-fourths of those suf- fering from tremor-dominant PD. Here we show that DBS does offer significant relief from PD-associated tremor in these areas, albeit at a slightly lower efficacy in the case of leg tremor. The relatively low incidence of severe chin tremors requires a larger study to conclusively characterize the relative impact of stimulation on this region.

The source of this difference in response is unclear. One possibility lies in the topography of tremor within the STN. Although overlapping to some degree, the somatotopic distribution of the leg classically lies medial to the arm within the STN. ${ }^{21}$ Thus, the differing response between arm and leg in subjects with equal baseline scores could reflect the specific localization of the lead within the target. ${ }^{2}$ However, this seems unlikely given the spread of stimulation across the relatively small dorsolateral motor aspect of the STN as well as the multiple overlapping somatotopic maps within the range of the electrical field. ${ }^{1}$ The fact that tremor reduction of the chin was not worse than arm or leg tremor reduction also points to the ability of STN stimulation to cover a broad somatotopic distribution. However, it is hard to exclude the possibility that a different subregion within the STN or neighboring structures might have proven superior for some tremors. Modeling the fields and spread of stimulation (e.g., involvement of proposed STN subloops or connections $)^{28,29,32}$ might allow for firmer conclusions to be drawn about differential targeting and stimulation parameters for arm versus leg tremors. Overall tremor improve- 
ment in the cohort still supports traditional targeting as sufficient in most cases.

Other neighboring structures, such as the posterior subthalamic area (PSA) or zona incerta (ZI), have been suggested as alternative targets for superior motor control, 6,7, $12,15,27,34$ yet we are unaware of any reports to date distinguishing between chin, arm, and leg tremor outcomes in these areas. Exploring differing outcomes in other brain targets used for management of PD tremor, such as the ventral intermediate nucleus (Vim) of the thalamus and globus pallidus internus (GPi), could add further insight into the generalizability of these findings, as could further exploration of other motor features, such as differing rigidity and bradykinesia responses following STN DBS. Alternatively, the presence of residual leg compared with arm tremor in equally affected limbs may also be simply a function of the mass of the leg versus the arm and/or a more proximal component, making it harder to control to the same degree. Leg tremors have been reported to persist despite functional improvement in cases of thalamic stimulation for orthostatic tremor as well, again perhaps pointing to the general challenge of controlling leg tremors irrespective of target or condition. ${ }^{8}$ The potential for leg response to differ from arm response should perhaps be broached with patients preoperatively, particularly if leg tremors are a prominent chief complaint.

There are a few limitations to our study that warrant consideration. The retrospective nature of our analysis coupled with some loss of incomplete data may bias the results. However, these variables are partly compensated by the relatively large data set in which the same experienced DBS team and rater were used throughout. Although the wide range of final follow-up periods (from 6 months to 4 years) captured by the data are another "real life" imperfection in our data analysis, it is mitigated by the previously reported observations that the effect of STN DBS on tremor can be maintained with little habituation or need for extensive reprogramming after the initial adjustment period. ${ }^{19}$ The possibility that non- or less-responsive patients might have attained improvement with different parameters cannot be excluded either, but all programming was undertaken by the same experienced programmer with multiple troubleshooting sessions for more challenging cases. These included exploration of more advanced programming options including interleaving, changes to constant current, and exploration of multiple contacts and parameters, as reflected in Table 1. Last, there may be inherent limitations of the UPDRS for tremor, which has been demonstrated to suffer from a ceiling effect and cannot capture finer gradations of improvement. Nevertheless, it has remained the standard measure of motor improvement despite these limitations. ${ }^{16}$

\section{Conclusions}

This retrospective single-center experience corroborates the ability of STN DBS to consistently and significantly improve PD-related tremor. More specifically, it sheds additional light on the characteristics of patients with tremor-predominant disease within a large PD cohort and examines the varying impact of stimulation on leg, arm, and chin tremor. No significant difference beyond average time to surgery distinguished DBS's impact on medication-responsive versus medication-nonresponsive tremors. Although all regions respond to stimulation, leg tremors did not as consistently improve to the same degree as arm tremors, even when both demonstrated a reduction compared with pre-DBS severity.

\section{References}

1. Abosch A, Hutchison WD, Saint-Cyr JA, Dostrovsky JO, Lozano AM: Movement-related neurons of the subthalamic nucleus in patients with Parkinson disease. J Neurosurg 97:1167-1172, 2002

2. Anheim M, Batir A, Fraix V, Silem M, Chabardès S, Seigneuret E, et al: Improvement in Parkinson disease by subthalamic nucleus stimulation based on electrode placement: effects of reimplantation. Arch Neurol 65:612616, 2008

3. Benabid AL, Chabardes S, Mitrofanis J, Pollak P: Deep brain stimulation of the subthalamic nucleus for the treatment of Parkinson's disease. Lancet Neurol 8:67-81, 2009

4. Bergman H, Wichmann T, DeLong MR: Reversal of experimental parkinsonism by lesions of the subthalamic nucleus. Science 249:1436-1438, 1990

5. Blahak C, Wöhrle JC, Capelle HH, Bäzner H, Grips E, Weigel R, et al: Tremor reduction by subthalamic nucleus stimulation and medication in advanced Parkinson's disease. J Neurol 254:169-178, 2007

6. Blomstedt P, Sandvik U, Tisch S: Deep brain stimulation in the posterior subthalamic area in the treatment of essential tremor. Mov Disord 25:1350-1356, 2010

7. Blomstedt P, Stenmark Persson R, Hariz GM, Linder J, Fredricks A, Häggström B, et al: Deep brain stimulation in the caudal zona incerta versus best medical treatment in patients with Parkinson's disease: a randomised blinded evaluation. J Neurol Neurosurg Psychiatry 89:710-716, 2018

8. Coleman RR, Starr PA, Katz M, Glass GA, Volz M, Khandhar SM, et al: Bilateral ventral intermediate nucleus thalamic deep brain stimulation in orthostatic tremor. Stereotact Funct Neurosurg 94:69-74, 2016

9. Deuschl G, Schade-Brittinger C, Krack P, Volkmann J, Schäfer H, Bötzel K, et al: A randomized trial of deep-brain stimulation for Parkinson's disease. N Engl J Med 355:896908, 2006

10. Dirkx MF, den Ouden HEM, Aarts E, Timmer MHM, Bloem BR, Toni I, et al: Dopamine controls Parkinson's tremor by inhibiting the cerebellar thalamus. Brain 140:721-734, 2017

11. Fasano A, Daniele A, Albanese A: Treatment of motor and non-motor features of Parkinson's disease with deep brain stimulation. Lancet Neurol 11:429-442, 2012

12. Fiechter M, Nowacki A, Oertel MF, Fichtner J, Debove I, Lachenmayer ML, et al: Deep brain stimulation for tremor: is there a common structure? Stereotact Funct Neurosurg 95:243-250, 2017

13. Fishman PS: Paradoxical aspects of parkinsonian tremor. Mov Disord 23:168-173, 2008

14. Ford B, Winfield L, Pullman SL, Frucht SJ, Du Y, Greene P, et al: Subthalamic nucleus stimulation in advanced Parkinson's disease: blinded assessments at one year follow up. J Neurol Neurosurg Psychiatry 75:1255-1259, 2004

15. Fytagoridis A, Åström M, Samuelsson J, Blomstedt P: Deep brain stimulation of the caudal zona incerta: tremor control in relation to the location of stimulation fields. Stereotact Funct Neurosurg 94:363-370, 2016

16. Goetz CG, Tilley BC, Shaftman SR, Stebbins GT, Fahn S, Martinez-Martin P, et al: Movement Disorder Societysponsored revision of the Unified Parkinson's Disease Rating 
Scale (MDS-UPDRS): scale presentation and clinimetric testing results. Mov Disord 23:2129-2170, 2008

17. Helmich RC: The distributed somatotopy of tremor: a window into the motor system. Exp Neurol 241:156-158, 2013

18. Katz M, Luciano MS, Carlson K, Luo P, Marks WJ Jr, Larson PS, et al: Differential effects of deep brain stimulation target on motor subtypes in Parkinson's disease. Ann Neurol 77:710-719, 2015

19. Krack P, Batir A, Van Blercom N, Chabardes S, Fraix V, Ardouin $\mathrm{C}$, et al: Five-year follow-up of bilateral stimulation of the subthalamic nucleus in advanced Parkinson's disease. $\mathbf{N}$ Engl J Med 349:1925-1934, 2003

20. Krack P, Benazzouz A, Pollak P, Limousin P, Piallat B, Hoffmann D, et al: Treatment of tremor in Parkinson's disease by subthalamic nucleus stimulation. Mov Disord 13:907-914, 1998

21. Lambert C, Zrinzo L, Nagy Z, Lutti A, Hariz M, Foltynie $\mathrm{T}$, et al: Confirmation of functional zones within the human subthalamic nucleus: patterns of connectivity and subparcellation using diffusion weighted imaging. Neuroimage 60:83-94, 2012

22. Lilleeng B, Gjerstad M, Baardsen R, Dalen I, Larsen JP: Motor symptoms after deep brain stimulation of the subthalamic nucleus. Acta Neurol Scand 131:298-304, 2015

23. Limousin P, Krack P, Pollak P, Benazzouz A, Ardouin C, Hoffmann D, et al: Electrical stimulation of the subthalamic nucleus in advanced Parkinson's disease. N Engl J Med 339:1105-1111, 1998

24. Limousin P, Pollak P, Benazzouz A, Hoffmann D, Le Bas JF, Broussolle E, et al: Effect of parkinsonian signs and symptoms of bilateral subthalamic nucleus stimulation. Lancet 345:91-95, 1995

25. Obeso JA, Olanow CW, Rodriguez-Oroz MC, Krack P, Kumar R, Lang AE: Deep-brain stimulation of the subthalamic nucleus or the pars interna of the globus pallidus in Parkinson's disease. N Engl J Med 345:956-963, 2001

26. Odekerken VJJ, van Laar T, Staal MJ, Mosch A, Hoffmann CFE, Nijssen PCG, et al: Subthalamic nucleus versus globus pallidus bilateral deep brain stimulation for advanced Parkinson's disease (NSTAPS study): a randomised controlled trial. Lancet Neurol 12:37-44, 2013

27. Plaha P, Khan S, Gill SS: Bilateral stimulation of the caudal zona incerta nucleus for tremor control. J Neurol Neurosurg Psychiatry 79:504-513, 2008

28. Reck C, Florin E, Wojtecki L, Krause H, Groiss S, Voges J, et al: Characterisation of tremor-associated local field potentials in the subthalamic nucleus in Parkinson's disease. Eur J Neurosci 29:599-612, 2009
29. Reck C, Himmel M, Florin E, Maarouf M, Sturm V, Wojtecki $\mathrm{L}$, et al: Coherence analysis of local field potentials in the subthalamic nucleus: differences in parkinsonian rest and postural tremor. Eur J Neurosci 32:1202-1214, 2010

30. Schuepbach WMM, Rau J, Knudsen K, Volkmann J, Krack P, Timmermann L, et al: Neurostimulation for Parkinson's disease with early motor complications. N Engl J Med 368:610-622, 2013

31. Tomlinson CL, Stowe R, Patel S, Rick C, Gray R, Clarke CE: Systematic review of levodopa dose equivalency reporting in Parkinson's disease. Mov Disord 25:2649-2653, 2010

32. Vanegas-Arroyave N, Lauro PM, Huang L, Hallett M, Horovitz SG, Zaghloul KA, et al: Tractography patterns of subthalamic nucleus deep brain stimulation. Brain 139:1200-1210, 2016

33. Weaver FM, Follett KA, Stern M, Luo P, Harris CL, Hur $\mathrm{K}$, et al: Randomized trial of deep brain stimulation for Parkinson disease: thirty-six-month outcomes. Neurology 79:55-65, 2012

34. Xie T, Bernard J, Warnke P: Post subthalamic area deep brain stimulation for tremors: a mini-review. Transl Neurodegener 1:20, 2012

\section{Disclosures}

Dr. Pourfar has received teaching honoraria (Medtronic, Inc., Teva Pharmaceuticals, US World Meds); consultation compensation (Abbott, Inc., Lundbeck Pharmaceuticals); and grant support (Boston Scientific, Inc.). Dr. Mogilner has received consultation compensation (Medtronic, Inc., Alpha-Omega Engineering, Abbott, Inc.) and grant support (Boston Scientific, Inc., Medtronic, Inc., St. Jude Medical, Inc.).

\section{Author Contributions}

Conception and design: Pourfar, Ko, Mogilner. Acquisition of data: Pourfar, Ko, Mogilner. Analysis and interpretation of data: Pourfar, Katlowitz. Drafting the article: Pourfar, Katlowitz. Critically revising the article: Pourfar, Katlowitz. Reviewed submitted version of manuscript: all authors. Approved the final version of the manuscript on behalf of all authors: Pourfar. Statistical analysis: Katlowitz. Administrative/technical/material support: Pourfar, Mogilner. Study supervision: Pourfar, Ko, Mogilner.

\section{Correspondence}

Michael Pourfar: New York University Langone Medical Center, New York, NY. michael.pourfar@nyumc.org. 